\title{
Efficiency simulations of thin film chalcogenide photovoltaic cells for different indoor lighting conditions
}

\author{
B. Minnaert ${ }^{1}$ and P. Veelaert ${ }^{1}$
}

\begin{abstract}
Photovoltaic (PV) energy is an efficient natural energy source for outdoor applications. However, for indoor applications, the efficiency of PV cells is much lower. Typically, the light intensity under artificial lighting conditions is less than $10 \mathrm{~W} / \mathrm{m}^{2}$ as compared to $100-1000 \mathrm{~W} / \mathrm{m}^{2}$ under outdoor conditions. Moreover, the spectrum is different from the outdoor solar spectrum. In this context, the question arises whether thin film chalcogenide photovoltaic cells are suitable for indoor use. This paper contributes to answering that question by comparing the power output of different thin film chalcogenide solar cells with the classical crystalline silicon cell as reference. This comparison is made for typical artificial light sources, i.e. an LED lamp, a "warm" and a "cool" fluorescent tube and a common incandescent and halogen lamp, which are compared to the outdoor AM 1.5 spectrum as reference. All light sources (including the outdoor spectrum) are scaled to an illumination of 500 lux to obtain a correct comparison. The best artificial light source for all cell types is the incandescent lamp which improves the performance of the cell up to a factor 3 compared with the AM 1.5 spectrum. One remarkable result is that a CdTe cell outperforms a CIGS cell with more than $33 \%$ in an indoor artificial lighting environment (except with an incandescent light source).
\end{abstract}

